\title{
Identidades estratégicas en los países de UNASUR y su impacto en la búsqueda de una identidad de defensa suramericana
}

\section{Identidades estratégicas nos países da UNASUR e seu impacto na busca de uma identidade de defesa sul-americana}

LUIS TIBILETTI*

\section{INTRODUCCIÓN}

El presente trabajo es continuación de mis reflexiones sobre la identidad estratégica publicadas en los Cuadernos de Actualidad en Defensa y Estrategia $\mathrm{N}^{\mathrm{O}} 4$ (Tibiletti 2009) y de mi ponencia en el X Congreso de la SAAP en Córdoba (Tibiletti 2011 ).

En momentos que los presidentes de UNASUR han requerido a través del Consejo de Defensa Sudamericano construir una identidad de defensa sudamericana y el Centro de Estudios Estratégicos de la Defensa (CEED) se encuentra trabajando en la identidad estratégica ${ }^{1}$, parecería válido acompañar lo más posible este camino desde la reflexión intelectual que permita entender las relaciones entre identidad nacional, identidad internacional e identidad de la defensa en el nivel regional, como es el mandato del $\mathrm{CDS}^{2}$, sin perder de vista el contexto de crisis mundial en el que nos encontramos y que ha revalorizado el papel de los estados.

En este trabajo entonces se pretende revisar el estado del debate sobre la construcción de las identidades nacionales, y dentro de ellas algunas subespecies como parecen ser la identidad internacional y la identidad estratégica a nuestro entender inescindible de ella.

Para ello procuramos llegar al concepto de identidad estratégica desde una visión que incluya una necesaria revisita del devenir epistemológico

\footnotetext{
* Profesor de la Escuela de Relaciones Internacionales de la Universidad del Salvador y de la Escuela de Defensa Nacional.
} 
en los estudios de las relaciones internacionales, los más específicos de seguridad internacional, los estudios estratégicos y las relaciones entre estos campos entre sí y la cada vez más necesaria vinculación de ellos con los estudios de la paz como objetivo normativo de la disciplina que consideramos fue olvidado en el transcurso de la segunda mitad del SXX.

\section{EL DEBATE SOBRE ESTRATEGIA Y SEGURIDAD EN LA REGIÓN: TEORÍA Y PRÁCTICA}

Esta concepción que desarrollaré es también producto de los largos años de reflexión desde una visión académica tanto en el Seminario de Seguridad Internacional y Cooperación para la Paz dictado junto a otros colegas (Gustavo Druetta, Marcela Donadío y Sebastián Muñoz) en la Escuela de Relaciones Internacionales de la USAL entre 1992 y 2004 como en el que dicto desde 2008 sobre Teoría y Práctica de la Construcción de la Paz con énfasis en América Latina en la misma casa de estudios. También desde la Cátedra de Defensa de la EDENA que integro desde 1994 y donde me especialicé inicialmente en seguridad internacional y políticas de defensa comparadas y desde 2008 en el Seminario sobre Política, Estrategia y Doctrinas de Defensa y donde continuamos junto a mi colega el Mag. Marcelo Marteletti con la indagación de cómo entender las culturas e identidades estratégicas.

En un plano mucho más de "operador de la realidad” la preocupación por las relaciones entre dos políticas públicas tan propiamente estatales como son la política exterior y la política de defensa, me llevó a constituirme tanto desde mi trabajo de asesor en el Congreso como de actor de la sociedad civil a través de una Ong (Seguridad Estratégica Regional- SER en el 200) en un permanente analista de las lógicas de sus pertinentes burocracias. Así pude comprender las difíciles relaciones con las sucesivas conducciones políticas de ambos ministerios en la búsqueda de definir las líneas maestras de una nueva inserción regional de la Argentina en materia de seguridad y defensa desde la reinstalación de la democracia. Pude percibir incluso el modo en que diferentes modos de entender la realidad de ambas burocracias terminan generando conflictos entre las conducciones políticas de las mismas.

Es desde esta experiencia de convivir treinta años entre diplomáticos que siempre prefieren autoreferenciarse como "grocianos" y estrategistas que no soportan otra clasificación que no sea la de "realistas" - desde Sun 
Tzu en adelante -, que surje el intento de encontrar nuevas preguntas explicatorias. Preguntas que sé necesariamente implican asumir una posición epistemológica y metodológica tanto para su formulación como para su indagación.

¿Cómo no preguntarse por ej. sobre el impacto de la relación entre diplomáticos y militares en un plano de las ciencias sociales, sin entender que más allá de las reflexiones filosóficas sobre la guerra y la paz en la historia de la humanidad, en tanto disciplinas sociales en sí el estudio de las relaciones internacionales y los asuntos estratégicos y de seguridad internacional comenzaron en momentos de diferente valoración de la racionalidad positivista?. Pero que además lo hicieron influídos por sus propios contextos que eran claramente diferentes a los de nuestras realidades latinoamericanas.

Resulta así la necesidad de nuevos aportes teóricos como una visión desde la sociología del conocimiento por ej. que permita clarificar como influyeron las contextos epistemológicos y las cuestiones histórico estructurales en la conformación de cada disciplina. Para poner un solo ejemplo: en la base epistemológica de las teorías explicativas - clásicas de todo pensamiento positivista - el mundo ES uno sólo y podemos entender sus reglas de funcionamiento mediante una correcta metodología, mientras que "la concepción constitutiva de la teoría no se compromete necesariamente con una relativización absoluta del mundo material, sino que cuestiona que ese mundo posea un significado dado por si sólo y autónomo del contexto de la interpretación". ${ }^{3}$

Así lo plantea en su último libro Barry Buzan, precisamente uno de los principales teóricos de la seguridad Internacional (Buzan y Hansen 2009, 35): "aunque hace bastante poco que la epistemología entró en debate en los estudios de seguridad internacional, ha sido algo muy influyente desde sus inicios".

El estudio científico de las relaciones internacionales comenzó a fines del SXIX en Europa donde ya había comenzado el declive del pensamiento positivista (basta recordar el efecto en la fe del progreso indefinido que tuvieron las primeras guerras con el uso de las modernas tecnologías y de allí surgieron las primeras Conferencias de la Paz) y tuvo luego su impulso a la luz del antirracionalismo más duro producto del horror de la 1ra Guerra Mundial; por eso la búsqueda de cómo evitar las guerras con una clara influencia del paradigma ideacional (y particularmente grociano en la relación entre naciones) y la fortaleza inédita exhibida por los movimientos 
pacifistas de la época. ${ }^{5}$

En este sentido el reciente trabajo de Lavallén sobre cómo el miedo al futuro (en especial el de los desarrollos tecnológicos bélicos) influyó en toda la literatura fantástica de Julio Verne y H.G.Wells - y persiste hasta hoy en las obras de Foucault -, constituye una mirada precisamente del tipo histórico-sociológica clave para entender la aparición de "ideas" como las de las Conferencia de la Paz y la Sociedad de las Naciones, entendida como esfuerzo supremo y superior por un orden que evitase el colapso de la humanidad (Lavallen 2012).

Contrariamente los estudios específicos de seguridad internacional que englobaron a los viejos estudios estratégicos y geopolíticos- nacieron en el ambiente de la 2 da posguerra acompañando el renacer del positivismo con fuerte énfasis realista especialmente en el mainstream centrado en las universidades y fundaciones norteamericanas.

Así lo afirman también Buzan y Hansen: "el impacto en los estudios de la seguridad internacional de haber nacido en los 40 con el reflujo neopositivista que impregna toda su producción” y siguen “ el concepto dominante de seguridad en los estudios de Seguridad internacional proviene de la visión realista de los Estudios Estratégicos" (Buzan y Hansen 2009, $21)^{6}$. Además como dice Walt crecieron al calor de los fondos otorgados a los investigadores y sus universidades por los departamentos de defensa de ambas superpotencias (Walt 1991, 236-239).

Como dicen Paradiso y Sebesta (2006),

"alentados por razones políticas, prosperan estudiosos de matriz realista, los cuales, según las pertinentes palabras de Waltz, no pretenden explicar todo sino algunas de las cosas más importantes. Como lo importante para los realistas es explicar el modo en que los estados se desempeñan en la arena internacional, dando por descontada, como elemento exógeno, la formación de sus intereses e identidad, se trata de una explicación que no hace más que justificar la realidad tal como la conocemos, legitimando la existencia de los estados y su utilización de la guerra como medio legítimo no sólo -y no tanto- para resolver las controversias, sino para actuar tout court en el mundo"

(Paradiso y Sebesta 2006, 3).

¿Devienen de este ethos realista clásico las siempre presentes invocaciones de los estrategistas por identificar los intereses del estado nación 
como "permanentes e inmutables"?. Basta con leer Clásicos de la Estrategia Moderna - escrita en 1944 - para confirmar estas afirmaciones:

La estrategia es el arte de controlar y emplear los recursos de una nación -o un conjunto de ellas- incluso sus Fuerzas Armadas con la finalidad de que sus intereses nacionales sean eficientemente mejorados y ofrezcan seguridad frente a enemigos efectivos, potenciales o simplemente supuestos. En tales asuntos la nación no puede convertirse en una sociedad contenciosa.

(Mead Earl 1968). ${ }^{7}$

¿Es por eso que reaccionan mal ante los planteos posracionalistas como por ej. los "constructivistas" que intentan entender los "intereses" como discurso y relaciones de poder cambiantes? ¿Hay por ello una tendencia natural entre los hombres más cercanos a "si vis pacem, parabellum” a entender la identidad nacional en clave esencialista? ¿Cómo influye esto en la definición de una identidad estratégica?. Si aceptamos sin más esta definición amplia y casi universal hoy de estrategia nacional ("arte de controlar y emplear todos los recursos para alcanzar los "intereses nacionales”) que la coloca al más alto nivel de la conducción política y a la vez su lógica es que en esos asuntos no hay contencioso posible: ¿cómo conviven en democracia las visiones plurales de la identidad - incluyendo la estratégica - ?.

Queda aquí un desafío para los politólogos de analizar la implicancia para el funcionamiento de un sistema político democrático de la sustracción del poder decisional que corresponde a las mayorías, en el marco del intercambio político y la negociación que son propias de los sistemas democráticos.

Según Martinez esto es "una auténtica declaración de guerra a la democracia misma, y fundamento viciado de la teoría de la seguridad nacional. Esa definición de estrategia realza el rol de las burocracias, olvidando que es la política la que pone los valores y los cambia así como se modifican las relaciones entre las clases, o como cambia la realidad local e internacional."

Por ello el debate "recién ahora cobra una mayor dimensión académica porque se ha asentado el tema de la paz en la región y el rechazo las versiones realistas dogmáticas que le han dado sustento a la estrategia como prescripción erga omnes". ${ }^{8}$ Más allá de las discusiones epistemológicas es interesante el impacto que estas ideas han tenido en la realidad de nuestros países. 
Así lo sostiene también Tapia Valdés quien basándose en Castoriadis desarrollará la relación entre Estrategocracia y seguridad nacional cuando dice

"el concepto de seguridad nacional creó una visión nueva de la estrategia, tradicionalmente centrada en el estudio de la guerra con sentido histórico y descriptiva, transformándola en prescriptiva y centrada en la producción de alternativas políticas; así la estrategia se ha convertido cada vez en una actividad de tipo político y con el concepto de "gran estrategia" pretende combinar las habilidades del soldado y el político..de allí que Kissinger diga "que la estrategia es el modo de sobrevivir de la sociedad"

(Tapia Valdes 1986).

Lo que el autor citado no llega a descubrir es que la definición de seguridad nacional que el toma como promedio de todos los documentos analizados en los dictaduras militares del Cono Sur es exactamente la misma noción que -como vimos-Edward Mearle había atribuído ya en 1944 a la estrategia: "la integración de la política exterior, interior y militar de un país con el propósito de coordinar el uso de sus potenciales político, económico, psicosocial y militar para garantizar el logro y subsistencia de los objetivos esenciales de la nación contra adversarios reales o potenciales, externos e internos”. Probablemente exija nuevas investigaciones profundizar sobre el significado esta sinonimia entre los conceptos de seguridad nacional y estrategia.

\section{LAS RELACIONES INTERNACIONALES Y LA IDENTIDAD}

El devenir de las teorías de relaciones internacionales y política exterior ha ido colocando en un lugar cada vez más relevante el tema de la identidad. Muy poco considerado inicialmente en las visiones del realismo clásico y el liberalismo basadas en consideraciones materiales o ideacionales y epistemologías positivistas, la aparición de los neo (realismo y liberalismo) donde lo que se discute es la estructura del sistema internacional y de su posterior síntesis neo-neo permitieron que aparecieran las primeras preocupaciones sobre el tema identitario.

Sin embargo fue la aparición de las visiones teóricas del constructivismo a partir de los 80 y las realidades de la posguerra fría las que colocaron poco a poco el tema en el centro de los debates, así como reapareció el interés por discutir más que el sistema internacional, la política exterior 
de los estados. ${ }^{9}$

De un primer esbozo que corría el riesgo de reemplazar la categoría que todo explica ${ }^{10}$ "interés nacional" ahora por "identidad nacional" con la misma indefinición sobre su constitución, los trabajos de fines de los 90 y de este siglo y la aparición del constructivismo crítico y las visiones histórico- estructurales como críticas a su vez del constructivismo extremo modificaron el panorama.

Así es que en el nivel de análisis de la política exterior aparece la categoría analítica de la identidad nacional como sujeto de estudio, tal como lo señala Merke:

"esta tesis sugiere que es tan necesario problematizar los intereses como las identidades. Esto implica demostrar cómo la identidad que configura la política exterior de un estado ha sido construida, cómo es comprendida y cómo esta comprensión da lugar a los intereses nacionales que guían la política exterior. Si la definición de intereses depende de la articulación de una identidad colectiva, entonces es necesario teorizar y estudiar empíricamente la construcción de las identidades y los procesos por los cuales estas identidades producen intereses." ${ }^{11}$

Bovero (1983: 31-57) dice que "la identidad colectiva es la idea de un bien o interés común que lleva a las personas a afirmar una identidad por semejanza, basada en una visión compartida de ese bien o interés común”.

Aquí toma sentido entonces el estudiar el proceso de construcción de la identidad nacional, ver el cómo y cuánto de esa identidad deriva de cómo se ha decantado socialmente el uso de lo militar en los distintos momentos de la historia del estado en cuestión; a eso es lo que denominaremos inicialmente como "identidad estratégica". ${ }^{12}$

\section{LA RELACIÓN ENTRE IDENTIDAD Y CULTURA}

\subsection{Filosofía y ciencias sociales: la transmodernidad}

Si hay algo que ha venido cambiando en las ciencias sociales en los últimos años es la necesidad de recuperar la relación con los aspectos más globales de los modos de conocer, tanto para trabajar en la propia disciplina como para relacionarse con otras.

Es por ello difícil hablar de "identidades" sin definir donde pararse en los debates filosóficos de esta época. Como dirá Casullo: "estamos atravesados, conformados y empantanados en la crónica de las discursividades modernas, de sus pasados radiantes y de sus supuestos y discutidos cre- 
púsculos actuales" (Casullo 1993, 62). ${ }^{13}$ Para no abundar en materias que otros desarrollan con mayor enjundia diré simplemente que frente a la segunda crisis de la modernidad que comienza casi al final del siglo corto de Hobsbawn y que dio en llamarse postmodernidad -por considerar esta crisis como terminal-, hay hoy una reacción que se expresa de diferentes maneras.

Así Larraín va a proponer “mirar contextuadamente y con énfasis sociológico estos debates" y va a hablar de "reflexividad racional” y sostener la necesidad de una “concepción histórico-estructural”, lo que luego aplicaremos a la identidad (Larraín 1996).

Por su parte hay quienes prefieren recuperar hoy el concepto de "transmodernidad”. Así en una buena síntesis de estos debates filosóficos-epistemológicos que elaborara para el IDICSO-USAL, Comini va a sostener que "la noción de transmodernidad pretende trascender la razón moderna -violenta, eurocéntrica , desarrollista y hegemónica- no por pura negación (como lo posmoderno) sino por incorporación de la alteridad (con base en Dussel)”, y además va a señalar como esta visión puede aplicarse al estudio del proceso de Unasur (Comini 2011).

\subsection{La construcción de las identidades particulares y colectivas: su relación inescindible con lo OTRO}

La construcción de las identidades particulares es un tema ampliamente debatido en las ciencias sociales. Larraín dirá que "las identidades personales y colectivas están interrelacionadas y se necesitan recíprocamente. No pueden haber identidades personales sin identidades colectivas y viceversa. Los individuos se definen por sus relaciones sociales y la sociedad se reproduce y cambia a través de acciones individuales. Las identidades personales son formadas por identidades colectivas culturalmente definidas, pero éstas no pueden existir separadamente de los individuos”. (Larraín 2001, 34).

Sin embargo el mismo autor nos advierte que la relación cercana no debe hacer olvidar las diferencias entre ambas identidades so pena de caer en el error del culturalismo (Mead, Benedit y otros) cuando quieren hablar de "carácter nacional " o "mentalidad de un pueblo”. Así afirma que "los rasgos psicológicos que se identifican como supuestamente pertenecientes a un carácter nacional muestran por sí mismos su inadecuación en la medida que claramente no son compartidos por todos los miembros de esas sociedades. Constituyen sobregeneralizaciones abstractas que no pueden 
predicarse a toda una nación...es un error ontologizar para un colectivo lo que son rasgos psicológicos particulares" (Larraín 2001, 36.).

Para toda la literatura de psicología social no existe identidad particular o colectiva que se pueda constituir sino es incluyendo la relación con el /lo OTRO. "La identidad presupone la existencia de OTROS, que tienen modos de vida, valores, costumbres e ideas diferentes. Así surge el nosotros distinto a ellos los otros", dirá el autor chileno que venimos siguiendo.

Lo mismo sucederá en el caso de la conformación de las identidades nacionales. Así por ej lo afirma Busso "La identidad se construye también en relación al otro, es decir que en el caso de las identidades nacionales tienen suma importancia las vinculaciones que los Estados establecen a nivel internacional con los diversos actores" (Busso 2010).

Lafer va a decir que "se puede hablar de identidades nacionales que, paradojicamente se formaron y se forman en función de la vida internacional, en el contacto y la interacción con el otro" (Lafer 2002, 1).

¿Cómo influye esa relación con lo otro en el caso de esa parte de la identidad nacional que denominamos estratégica?. Si aceptamos que en la base de ella está el modo de entender el uso de lo militar en su historia y tal como vimos que Earl definía a la estrategia frente al "enemigo efectivo, potencial o supuesto" entenderemos cómo juega aquí la alteridad: "Si bien la diferenciación es un proceso indispensable también puede constituir un peligro si la exacerbación de las diferencias conduce a la hostilidad" (Larraín 2001, 32).

Así para una mirada "estratégica" la alteridad va ser siempre más fuertemente considerada en términos de diferenciación negativa, tendiendo siempre al extremo del conflicto y nunca de la cooperación SALVO que aparezca el enemigo común y allí aparece la alianza, pero siempre temporal mientras dure la amenaza común.

Al menos así será mientras no se produzca la aparición de una corriente de "estrategistas críticos" que pueda incluir otras miradas epistemológicas tal como sucedió por ej en los asuntos de geopolítica, seguridad internacional y estudios de la paz.

\subsection{Cultura e identidad, cambio y continuidad}

¿Cuál es la relación entre cultura e identidad en un plano conceptual? ¿Cuál de ellas es más permeable en el tiempo?.

Hay muchísimas posiciones en la literatura al respecto tanto en el plano 
individual como cuando transferimos estos conceptos a lo colectivo, como en el caso de las comunidades nacionales.

Así por ejemplo dos autoras cubanas sostendrán que "la identidad es producto de los avatares de la cultura a lo largo del proceso histórico”. (Baeza y García 1996).

Wallerstein va a decir que: "En primer lugar, las múltiples comunidades a las que todos pertenecemos, cuyos "valores" adoptamos, hacia las cuales expresamos "lealtades", que definen nuestra "identidad social” son todas, todas y cada una de ellas construcciones históricas. E incluso, más importante aún, están sometidos a permanente reconstrucción" (Wallerstein 1991).

Larraín sostiene que la cultura es más permeable que la identidad que es más resiliente y por eso no debemos tenerle miedo a las influencias culturales en épocas de globalización y gran influencia externa porque no todos esos cambios finalmente modificaran la identidad” (Larraín 2005). ${ }^{14}$

Parece también útil recordar - tal como lo hace Busso en un reciente trabajo $^{15}$ - a Castells cuando señala que "la construcción social de la identidad se desarrolla en un contexto de relaciones de poder" y por lo tanto en el caso de la "identidad nacional" habrá una influencia del lugar que ocupe ese colectivo en el entramado internacional del poder. Por ello serán también los vaivenes en ese entramado los que jueguen en el proceso de construcción/reconstrucción de la identidad.

\section{LAS DISTINTAS VISIONES DE LA IDENTIDAD LATINOAMERICANA}

Son muchos los autores que han tratado el tema de la identidad latinoamericana a lo largo de los dos siglos desde la independencia. Así lo señala uno de los mayores analistas argentinos del tema: "la idea latente de una gran nación americana exhibe una tradición teórica y activa desde los tiempos de la independencia, ha sido sostenida por diversos expositores y corrientes cuya divulgación ha dado lugar a un vastísimo corpus literario y político junto a una exégesis no menos frondosa y a largos desvelos generacionales" (Biagini 2009, 28).

Sin embargo los principales cuestionamientos acerca del ¿qué somos?, aparecen siempre ligados a contextos de crisis, en particular las crisis de la modernidad a caballo de los cambios de siglo; pero además como bien señala Casullo en una "modernidad descentrada que agolpó en un mismo espacio y tiempo irrupciones industrialistas y testimonios de mundos in- 
dígenas, seducción y saqueo de los poderes extranjeros, desacoples profundos entre sus culturas populares y las racionalizaciones dominantes" (Casullo 1993, 62). Aquí se advierte con claridad lo que decíamos antes de cómo la construcción de la identidad se lleva a cabo en relaciones de poder estructurales.

Es por eso que Larraín va a decir que hay dos grandes posiciones frente al tema de la relación entre identidad y modernización: quienes siempre han propuesto una identidad de raigambre europea que acompañe el proceso de "modernización" y quienes procuran rescatar y poner en valor una identidad propia aún a costa de la modernidad. (Larraín 1996)

Seguimos nuevamente a Larraín, (op.cit 2001) cuando dice "que en América Latina ha existido siempre una conciencia de identidad latinoamericana, articulada con las identidades nacionales". Luego describe las cinco tendencias interpretativas de la identidad latinoamericana que se han verificado en la literatura y las clasifica en:

a) Corrientes hispanistas

b) Corrientes indigenistas

c) Mestizaje cultural

d) Mestizaje religioso

e) Corriente de desintegración y búsqueda de identidad

También sostiene que todas ellas tienden a una visión esencialista ya sea en clave positivista o incluso posmodernas por lo que va a proponer su alternativa histórico-estructural que luego desarrollaremos.

Si es importante señalar ahora a los fines de nuestro trabajo que en ese proceso de construcción de la identidad latinoamericana ha jugado siempre un papel muy fuerte la relación de la identidad nacional con la latinoamericanidad. Es decir que "cada identidad nacional en AL tiene así un componente común latinoamericano y otro componente específico” y que aquí hay claramente diferencias en cómo se procesó esta relación en los casos de los pueblos "testimonios", "nuevos" o "trasplantados" para seguir a Darcy Ribeiro citado por Larraín (op.cit 2001).

\subsection{La larga paz sudamericana: ¿una señal de identidad?}

Más próximo a nuestro objetivo del trabajo resulta necesario mencionar las características de la latinoamericaneidad vinculadas a la política exterior, que van a proponer Paradiso y Luna Pont. ${ }^{16}$ 
En breve síntesis ellos señalan los siguientes elementos de la condición americana de nuestra región:

- Idea de unidad expresada desde el proceso libertario con presencia permanente de pulsiones por la integración que se expresan en las áreas culturales y también políticas;

- El status periférico al orden internacional también definido como la "periferidad";

- La “cohabitación” con un claro poder hegemónico que llevará a tensiones permanentes entre mantenerlo cerca pero limitar su intervencionismo desorbitado;

- Una cultura política compartida;

- Un sustrato de “nacionalismo integrador” (la permanente mención a la Patria grande);

- Fuerte preeminencia del dilema del desarrollo antes que del de la seguridad.

Todo esto es lo que permite luego verificar la clara preeminencia de la cooperación antes de que el conflicto en la región y es lo que expresaran de diferentes maneras autores como Kacowitz o Holsti. ${ }^{17}$

En cuanto a lo suramericano el concepto de la "larga paz sudamericana” aparece en los trabajos de Kacowitz en los 90 (Kacowitz 1996) y luego va a ser retomado por otros autores. Siendo ahora claramente normativos consideramos que esta es una de las claves de nuestra identidad a trabajar muy fuertemente desde los organismos de integración como UNASUR, el Consejo de Cancilleres y el de Defensa.

\subsection{Las distintas visiones de la identidad nacional. La opción histórica-estructural, sus implicancias: la pluralidad de visiones y la identidad como proyecto}

Como decíamos Larrain (op.cit 2001) va a decir que hay - tanto de la identidad latinoamericana como de las nacionales tres visiones diferenciadas: las visiones "esencialistas" acentúan la importancia de lo étnico-cultural, ya sea lo español, lo indígena o lo racional y rechazan las mezclas y la 
evolución histórica "fijan la identidad cultural en el período histórico fundacional o en categorías trascendentales y se niegan a aceptar contribuciones de otras épocas o categorías". "Al considerar la identidad como una esencia inmutable, el esencialismo descuida la historia y el hecho comprobable de que la identidad va cambiando".

Por otra parte va criticar también las visiones constructivistas extremas que pretenden negar que existan esencias y racionalidad y limitan todo al "discurso" construido desde arriba en la esfera pública "descuidando el análisis de las formas discursivas y de las prácticas populares y privadas”.

Por ello desde su opción por la "reflexividad racional" como superadora del debate modernidad-posmodernidad va a plantear una tercera opción alejada de esos extremos que llama "histórico-estructural".

En ella la identidad "es algo que está en permanente construcción y reconstrucción dentro de nuevos contextos y situaciones históricas, algo de lo que nunca puede afirmarse que está finalmente resuelto o constituído como un conjunto fijo de cualidades, valores y experiencias comunes. Por otra parte no concibe esa construcción sólo como un proceso discursivo público sino que considera las prácticas y significados sedimentados en la vida diaria de las personas".

Afirma también que siempre hay varias versiones públicas de la identidad en competencia y las personas no se entregan pasivamente a ellas sino que "mantienen su capacidad crítica para discriminar, aceptar esas ofertas de identidad que finalmente no existe en una versión determinada, por sí misma, por comprensiva y atrayente que sea sino que existe, más bien, en la relación dinámica entre los diversos discursos identitarios con el autoreconocimiento efectivo de la gente en sus prácticas”.

Podemos agregar a Alexander Wendt cuando dijo que "cambiando las prácticas cambiará el conocimiento intersubjetivo que constituye las identidades" (Wendt 1994).

Aquí es donde tendría sentido que se investigue hasta qué punto los cambios en las prácticas de interrelación de los estados por ej del Cono Sur en términos de cooperación y conflicto desde la recuperación de los regímenes democráticos ha decantado en transformaciones de sus identidades estratégicas. Por lo que se lee en los libros blancos y las discusiones "estratégicas” parecería que se mantiene una dualidad en términos de mantener la disuasión entre estados que buscan integrarse cada día más. Algunos incluso han llamado a esto la "disuasión cooperativa".

Si nos colocamos frente a un objetivo como es el de la integración pro- 
clamada por UNASUR, resulta clave recuperar la visión de la identidad como también un proyecto de futuro tal como lo hace Habermas "la identidad no es algo ya dado, sino también y simultáneamente nuestro propio proyecto" (Habermas 1992, 243). En este proyecto de futuro, ¿puede la estrategia hacer un lugar a la paz?

\section{EL CONCEPTO DE CULTURA ESTRATÉGICA EN LOS ESTUDIOS DE SEGURIDAD INTERNACIONAL Y LA OPCIÓN POR IDENTIDAD ESTRATÉGICA COMO CATEGORÍA DE ANÁLISIS}

De acuerdo con Sondahus (2006) el concepto de cultura estratégica aparece por primera vez en la literatura en un informe de Jack Snider para la Rand Corporation en 1977; así lo recoge Menezes Teixeira Jr (2011) y agrega la definición del concepto como "la suma total de ideas, respuestas mentales condicionadas y patrones de comportamiento habituales que los miembros de una comunidad estratégica nacional adquieren a través de la educación o la imitación”.

Podemos ver aquí la idea de que la cultura estratégica no es algo de la sociedad o del estado sino una propiedad asignada a los "miembros de la comunidad estratégica nacional” ¿Qué sentido tendrá esta afirmación cuando hablemos de cambios en la cultura estratégica?

Alistair Jhonston va a utilizar la definición de cultura del antropólogo Geertz para definir a la cultura estratégica como un "sistema de símbolos que actúa para establecer las preferencias estratégicas generalizadas y de larga duración mediante la formulación de conceptos de la función y eficacia de la fuerza militar en relación de un estado a otro en los asuntos políticos y que así reviste a estas concepciones con un aura de objetividad que hacen que esas preferencias estratégicas parezcan singularmente realistas y eficaces" (Johnston 1995, 46).

Continuando con Teixeira Jr., este autor brasilero va a sostener que "la necesidad de un cambio en la cultura estratégica de la región enmarcada en la creación de una identidad suramericana en materia de defensa encuentra eco en los aspectos institucionales y objetivos del Consejo de Defensa Suramericano". Luego de analizarlos en detalle va a concluir -coincidiendo con nuestras consideraciones al respecto- en que “ el CDS se deberá consolidar como una institución de gerenciamiento de la defensa pero también incluyendo la promoción de la paz y la solución pacífica de controversias”.

Tomando como base lo que señaláramos sobre la relación entre cultura e 
identidad parece más adecuado para nuestro análisis considerar la categoría "identidad estratégica" pues está es la que es posible ayudar a "construir" en el nivel regional tal como lo ha pedido la conducción de UNASUR.

De esta manera la identidad estratégica vendría a ser la foto de lo que hoy ha decantado a nivel nacional de cada cultura estratégica y en el diálogo entre ellas los esbozos posibles de dibujar de la foto actual suramericana.

\subsection{Elementos a considerar en la identificación de una identidad estratégica nacional}

Decíamos que se considera a la Identidad Estratégica Nacional (IEN) como aquellos elementos decantados de los vaivenes de la cultura estratégica que definen el modo en que una comunidad organizada en un determinado estado nación se ha relacionado con el uso de la violencia armada para el logro de sus objetivos nacionales a lo largo de su historia.

Si bien esta relación puede surgir del análisis objetivo de elementos históricos también tiene gran influencia en la misma la percepción que esa comunidad ha construído (el relato) de su propio pasado y muy en particular de la etapa fundacional del estado -nación considerado.

Influyen en esa construcción de la identidad, elementos materiales dados en primer lugar por la geografía política, tales como el tipo de uso y la valoración de los distintos componentes de su territorio y las características geoestratégicas del mismo (entendidas como las fortalezas y vulnerabilidades físicas para su defensa). Luego aparece claramente la influencia de un conjunto de consideraciones antropológicas, sociales, culturales, religiosas, ideológicas y políticas (en este último caso por ejemplo, el tipo de régimen).

Si bien también se advierte en esta construcción el impacto que provoca el modo de relación a lo interno del estado con la manera de ejercer el monopolio de la violencia, el elemento fundamental en la construcción de la IEN está dado por la relación sostenida en primer lugar con los vecinos y luego con los demás actores a nivel subregional, regional y global.

De todo este complejo proceso uno puede en un momento determinado sacar una foto que identificase los elementos principales constitutivos de la IEN del actor considerado y preguntarse:

Este estado-nación:

¿Cómo entiende el funcionamiento del sistema internacional, regional y 
su entorno estratégico en términos de cooperación-conflicto?

¿Cómo se ve a sí mismo dentro de ese entramado y en esos términos?

¿Cuáles son aquellos intereses propios o compartidos por los qué está dispuesto a recurrir a la violencia armada para defenderlos?

¿De quién piensa que deberá defenderse?

¿Con quienes se imagina cooperando para defenderse?

¿Con qué y cómo piensa defenderse?

Por supuesto que también cada una de estas preguntas podrían formularse con expresiones más formales propias del método de planeamiento estratégico tales como intereses nacionales, intereses vitales, intereses estratégicos, etc , pero se han usado estos conceptos más amplios para acercarse a la idea de que esto es una construcción social a la que por lo general una gran parte de la sociedad contribuye y alejarlos de esa visión hiperrealista de los intereses permanentes al que nos referíamos como característica de los estudios estratégicos. Además estas preguntas debe agregarse la indagación sobre los elementos ideacionales y de prácticas cotidianas que construyen las propias percepciones en tanto aprendizajes que construyen identidades tal como diría Wendt (op.cit).

También la lectura deberá incluir las muy diferentes visiones al interior de la comunidad que como veíamos en la consideración de la identidad nacional se mantienen presentes, desaparecen, reaparecen, se enfrentan y se reconstruyen además en permanente diálogo con los otros estados en particular los vecinos de la subregión, como ya pasó desde mediados de los '80 en el Cono Sur (Tibiletti 2009).

\section{UNASUR, CDS Y EL DESAFÍO DE LA CONSTRUCCIÓN DE UNA IDENTIDAD ESTRATÉGICA REGIONAL}

El problema inicial para definir una Identidad Estratégica Regional (IER) es definir el criterio para considerar a un grupo de estados- nación como Región. Hay mucha discusión sobre ello, pero lo interesante para nuestro análisis es que precisamente uno de esos criterios puede ser precisamente que compartan una IER.

Durante los finales de los 80 y los 90 había una discusión muy grande sobre el contenido del concepto Cono Sur. ¿Como incluir en el Cono Sur se decía a un Brasil que limita al oeste y noroeste con Peru, Colombia y Venezuela, tres miembros de los más activos de la Comunidad Andina de 
Naciones?. Y fue el principio de la IER lo que resolvió el asunto. Toda la construcción del Mercosur como Zona de Paz- incluyendo a Chile en tanto miembro no pleno del grupo-, permitió identificar a un grupo de países que decidieron cambiar su modo de relación estratégico histórico. Así se pasó de las hipótesis de guerra cruzadas con ejes geopolíticos entre Santiago y Brasilia vs Lima-Buenos Aires y el juego de la atracción hacía cada uno de esos ejes para los otros estados de la zona (Bolivia, Paraguay y Uruguay), a la lógica de la cooperación, las medidas de confianza y la construcción de un espacio de seguridad cooperativa.

Finalmente se aceptó que la región Conosur tuviera existencia considerando en ella la dimensión platina del Brasil y eso le permitió a este país rediseñar su estrategia de defensa considerando cualquier hipótesis de guerra en su costado sur como una situación de catástrofe que debía ser evitada.

De tal modo definida una región podemos buscar una serie de datos que nos ayuden a identificar la IER, por ejemplo:

- Conflictos entre los estados de la región y su modo de resolución en el tiempo;

- Grado de disposición entre los estados de la región a alentar la participación de sus pares en la resolución de sus conflictos bilaterales y efectividad de dicha participación cuando se haya dado;

- Historia de las tentativas de construcción de IER y su vigencia en la percepción -de los pueblos;

- Relaciones con otras regiones de los principales estados o intentos de relación desde la región;

- Influencia de la región en las relaciones estratégicas regionales (en este caso obviamente las interamericanas) y las globales (peso de la región en el sistema ONU).

Los cambios que trajeron consigo los procesos de transición a la democracia a partir de mediados de los '80 modificaron las culturas estratégicas y así en algunos casos - como el de Argentina - (Tibiletti 2009), podemos afirmar que tuvieron un fuerte impacto en la región y permitieron reflotar los elementos identitarios mencionados por Paradiso/Luna Pont y que la guerra fría había sólo disimulado.

Así por ejemplo: 
- Chile y Uruguay abandonan poco tiempo después que Argentina los elementos conceptuales de la DSN. Esto es más lento en Brasil pues ellos construyeron una versión propia de la DSN ligada a su modelo desarrollista y las características más estructuralmente elitista del modelo de articulación que nace en el varguismo y se mantendrá por 60 años hasta la llegada de Lula (me refiero al triángulo de poder FIESP, FFAA, Itamaraty que sostendrá el modelo de desarrollo dejando un papel menor al sistema político representativo)

- Los Ministerios de Defensa del Cono Sur van gradualmente avanzando hacia una mayor capacidad de conducción política de las FFAA no sólo por menor resistencia de éstas sino por una creciente capacidad y disposición de sectores civiles y políticos para asumir la tarea.

- Creciente articulación entre los Ministerios de Defensa del Cono Sur para el desarrollo de actividades de cooperación e incluso de articulación de posiciones frente a reuniones de carácter regional como las Conferencias de Ministros de las Américas.

- En algunos países varios autores señalan la existencia de una crisis de identidad en sus respectivas Fuerzas Armadas; así por ej. Lopez y Saín para el caso argentino y Soares y Rizzo de Oliveira para el brasilero.

Cabría entonces preguntarse ¿cuántos de estos cambios en las culturas estratégicas y las crisis de identidades han devenido en un cambio en la identidad estratégica de esos países?.

Por lo que señalábamos que circula en los Libros Blancos, en los Encuentros de Estudios Estratégicos por ej de Brasil y Argentina o el $1 \mathrm{er}$ Encuentro Suramericano de Río de Janeiro en 2009 y en otros muchos otros foros de reflexión de los asuntos estratégicos, pareciera que dicha modificación no ha alcanzado un grado significativo o por lo menos no ha sido autopercibida por los actores "dueños" del "pensamiento estratégico" en la región. ¿Qué sucede entonces?.

Creo que podríamos aquí retomar lo indicado en nuestra introducción: el peso de la cuestión de la autonomía militar en la región y la debilidad de las estructuras de conducción política. Decía por ej Rizzo de Oliveira (2005) refiriéndose al caso de las FFAA de Brasil durante la transición: “o aparelho militar aparece mais como externo ao sistema democrático decisório do que como parte de ele (...) tendendo a construir una identidade por oposiçao aos centros decisórios”.

Colocar esta variable claramente interviniente nos permitirá entonces 
discurrir sobre el mentado objetivo para el CDS y el CEE de la construcción de una identidad estratégica suramericana.

\section{CONCLUSIONES}

Veamos un rápido resumen de lo que hemos planteado hasta aquí para luego elaborar una conclusión:

- Las identidades colectivas son siempre cambiantes, en permanente reconstrucción y en ello juega mucho la relación con el otro colectivo y tiene un componente de proyecto común;

- Para el pensamiento estratégico de los militares anclado en el realismo clásico la base es la desconfianza en el otro como síntesis de toda la historia de la humanidad;

- Toda identidad colectiva tiene en permanente disputa varias visiones sobre ella misma y la tensión entre ellas también es parte de esa identidad;

- Para los estrategistas - civiles y militares - en el posicionamiento estratégico de un país no puede haber lugar a “controversias” sobre quién es el enemigo y cómo enfrentarlo o sea no puede haber más de UNA identidad estratégica;

- La "burocracia diplomática" no suele ser fácil de orientar hacia los objetivos de los conductores políticos democráticos por varias razones pero especialmente por su supuesta posesión de "conocimiento" histórico y de la "racionalidad diplomática” frente al "voluntarismo” político;

- La "burocracia militar" por su parte tiene en la región un alto grado de historia “autonómica” y los órganos de conducción de la política o sea los Ministerios de Defensa son bastante débiles tanto en sus atribuciones como en la capacidad de ejercicio de las mismas.

¿Qué imaginamos sucederá si en estas condiciones le exigimos a un órgano como el CDS constituído por los Ministerios de Defensa que desarrollen una identidad estratégica suramericana?.

Podría suceder que los Ministerios de Defensa procedan a delegar la tarea en el Centro de Estudios Estratégicos de la Defensa (CEED); el mismo se completará prioritariamente por cuadros militares que repetirán sus apreciaciones estratégicas habituales y luego formularan una propuesta de identidad basada en todos los clichés del pensamiento estratégico ya ana- 
lizado. Los civiles que puedan concurrir a la tarea podrán cuando más formular inteligentes intentos como el reciente de un académico brasilero -el Dr Hector Saint Pierre-, en la conferencia inaugural de un seminario del CDS de Ecuador (Mayo 2012) para tratar de mantener los objetivos de las conducciones políticas forzando el léxico de los estrategistas para hablar de "disuasión cooperativa”, lo que podría quizás ser el primer intento de formulación de una rama de la estrategia crítica.

Fue previendo este natural desenlace que un grupo numeroso de académicos cuando estaba en construcción el proceso del CDS reclamábamos que el mismo estuviese integrado por partes iguales por funcionarios de defensa y cancillerías, con la participación de un delegado directo de los presidentes y con un fuerte contacto con el mundo académico relacionado con la temática. ${ }^{18}$

Sólo así se podrá construir como proponíamos más arriba una identidad de defensa suramericana que recoja lo más útil para nuestros pueblos que es precisamente esa "larga paz sudamericana” y también comenzar a debatir un nuevo sentido de lo "estratégico" y de la "seguridad regional" pero con la impronta de ser parte de una identidad como proyecto político. ${ }^{19}$

Creemos que sólo el análisis detallado de cómo cada país de nuestra subregión construyó su identidad estratégica en un juego permanente de percepciones, intereses de sector y realidades concretas de amenazas a su independencia y la lucha histórica por mayores grados de autonomía, permitirá delinear los modos para una nueva construcción acorde a los intereses reales de nuestra región en el siglo XXI, que sin duda pasan por mantener nuestro espacio de paz. 


\section{NOTAS}

1. Para la reflexión sobre cultura e identidad estratégica tengo que agradecer particularmente al Almte Fernando García quien me aportó un primer esbozo de cómo entenderlas, así como a todos los demás directores de SER en el 2000 en especial a Jaime Garreta y Juan Carlos Melián. Más tarde compartí con el primer de Secretario de Asuntos Exteriores de Defensa José M. Vazquez Ocampo y con su sucesor Alfredo Forti las preocupaciones por la construcción de una identidad regional de defensa.

2. Artículo 4(b) del Estatuto del CDS donde se especifica que uno de los objetivos

"es construir una identidad suramericana en materia de defensa". http://www. unasurcds.org

3. Patrone, César. 2012. "Neoinstiucionalismo y síntesis teórica".

4. Traducción del autor.

5. Paradiso José, entrevista con el autor. Él también explica como los elementos estructurales - la 1ra globalización y la modificación de los equilibrios de poder en Europa más la aparición de dos nuevas potencias como EEUU y Japón - influyeron en los primeros análisis de las RRII más allá de las cuestiones epistemológicas en las que se centró el análisis en este artículo.

6. Traducción del autor.

7. Esta afirmación resaltada constituye la base del pensamiento autoritario ya no sólo de los estrategistas sino también de los "estrategócratas" que fundamentaron todas las dictaduras militares de Suramérica en los años de la Guerra Fría. Implica que todo aquel que discrepa con la caracterización del "enemigo" hecha por los especialistas "realistas", es en sí un enemigo de la nación. El libro mencionado se utilizo como base de la formación en estrategia de los oficiales del Ejército Argentino al menos en la década de los 60 .

8. Dr. Pablo Martínez Director Comisión de Defensa Senado de la Nación, y profesor de la Escuela de Defensa Nacional , Argentina, entrevista con el autor, 22/6/2012.

9. Lapid, Yosef y Kratochwil, Friedrich, eds. 1996, The Return of Culture and Identity. In: IR Theory. Colorado: Lynne Rienner.

10. Algunos autores utilizan para este proceso el verbo "reificar" pero el mismo no existe en el diccionario de la RAE.

11. Merke, Federico. 2004. "Identidad y política exterior en teoría de las Relaciones Internacionales”, IDICSO, USAL, Bs As. 
12. Recientemente descubrí durante una entrevista con un doctorando checo que en algunas universidades europeas -como las de su país-, se diferencia entre identidad de defensa - entendida como expresión sedimentada de las Políticas de Defensa de un país en su historia- e identidad estratégica considerando allí estrictamente lo militar. Si bien en términos teóricos tal distinción es válida nos volvemos a encontrar con la dificultad de aplicarlos en una región como la nuestra donde NUNCA en el último siglo los políticos -con excepción de los que provenían del ámbito militar-, ni los sistemas políticos como tales representados en el Congreso fijaron sus políticas de defensa y delegaron tal tarea en los propios militares lo que llevó a la confusión de los ámbitos político y estratégicos en materia de defensa.

13. CASULLO Nicolás, "El debate modernidad-posmodernidad", Ed. Cielo por Asalto, Bs As 1993.

14. Reportaje de Peralta Gainza, Patricia , CLAES-D3E , 7/9/2005, Montevideo.

15. Busso, Anabella 2010. "Fuerzas profundas e identidad: su impacto en la política exterior".

Universidad Nacional de Rosario.

16. Seguimos en esto a Paradiso José y Luna Pont Mariana, "Paz y guerra en la trayectoria latinoamericana", Revista Universidad e Integracion, Estudios y Documentos sobre América Latina y el Caribe, INTAL, 1/03, Bs As 2003.

17. Holsti, K. J.; 1996. The state, war, and the state of war. Cambridge: Cambridge University Press. Capítulos 2 y 7. Kacowicz, Arie. Latina America as an International Society: a Grotian variation of regional Order and Community. Instruments of Análisis, Puente Europa 52.

18. El documento puede consultarse en www.unsurcds.org entrando en opiniones y luego en Centros y ONGs, última visita 6/7/2012.

19. Un autor canadiense ha afirmado incluso que los estudios estratégicos deben incluir "el aporte y la influencia de las instituciones internacionales y regionales de seguridad en la promoción del multilateralismo y la cooperación provocando con toda probabilidad, la evolución del sistema internacional hacia regímenes de seguridad basados en el derecho y no el poder y la aplicación de la fuerza (esencialmente militar)", David Charles Philippe. 2008. Los campos de investigación de los estudios estratégicos: la guerra y la paz". Madrid: FRIDE. 


\section{LAS DISTINTAS OFERTAS DE IDENTIDADES ESTRATÉGICAS EN LOS PAÍSES DE UNASUR Y SU IMPACTO EN LA BÚSQUEDA DE UNA IDENTIDAD DE DEFENSA SURAMERICANA}

\section{RESUMEN}

La decisión de los presidentes de la región de promover la construcción de una identidad de defensa suramericana pone nuevamente en debate los temas identitarios tanto a nivel regional como nacional. En este terreno - donde en el pasado eran hegemónicas las visiones esencialistas que consideraban a la identidad como algo nacido de una vez e inmutable -, hoy se le oponen otras visiones incluyendo alguna proveniente de un constructivismo extremo, donde casi no se reconocerían huellas en el pasado y todo depende de quien pueda imponer su "relato". Por ello en el presente artículo se propugna el paradigma histórico-estructuralista como superación del contrapunto y define a una parte de esa identidad nacional a la que denomina estratégica. Asimismo desarrolla que siempre existen - en ambos niveles nacional y regional -, identidades estratégicas en tensión y que ellas tienen diferentes impactos tanto en el logro de los objetivos asociativos como en el mantenimiento de la larga paz sudamericana.

Palabras-clave: UNASUR. Identidad de Defensa. Identidades Estratégicas. Identidad Nacional.

\section{REFERÊNCIAS}

Anderson, Benedict. 1993. Comunidades imaginadas: una reflexión sobre el origen y la difusión del nacionalismo. México: Ed. Fondo de Cultura Económica.

Biagini, Hugo. 2009. Identidad argentina y compromiso latinoamericano. Colección Humanidades y Artes, serie Filosofía. Lanús: Edic. UNLA.

Bovero, Michelangelo. 1983. Identitá individuali y colletive. Milán: Richerche. 31-37

Busso, Anabella. 2010. Fuerzas profundas e identidad: su impacto en la política exterior. Universidad Nacional de Rosario.

Buzan, Barry, y Hansen, Lene. 2009. The evolution of International Security Studies $(I S S)$. London: Cambridge University Press. 
Casullo, Nicolás. 1993. El debate modernidad-posmodernidad. Bs As: Ed. Cielo por Asalto.

Comini, Nicolás. 2001. Combatiendo la glosa: escolástica y transmodernidad en los estudios sobre Unasur. Usal: Jornadas IDICSO.

Holsti, K. J. 1996. The state, war, and the state of war. Cambridge: Cambridge University Press. Capítulos 2 y 7.

Johnston, Alistair. 1995. Thinking about strategic culture. Vol. 19. Spring: International Security.

Kacowicz, Arie. 2006. Latina America as an International Society: a Grotian variation of regional Order and Community. Nro 5. Revista Puente@europa. Buenos Aires: Edic. Universitá di Bologna Representación.

Lafer, Celso. 2001. La identidad internacional de Brasil. México: FCE.

Larraín, Jorge. 1996. Modernidad, razón e identidad en América Latina. Santiago de Chile: Ed. Andrés Bello.

Larraín, Jorge. 2000. Elementos teóricos para el análisis de la identidad nacional y la globalización. In: ¿Hay patria que defender?. Santiago de Chile: Ediciones del segundo centenario. CED.

Larraín, Jorge. 2001. Identidad Chilena. Colección Escafandra. Santiago de Chile: Ed. LOM.

Mead Earl, Edward. 1944. Creadores de la Estrategia Moderna. Vol. 1. Princeton University Press.

Mead Earl, Edward. 1968. Creadores de la Estrategia Moderna. Bs As: Ed. Círculo Militar.

Meneses Teixeira, Jr. Augusto Wagner. 2011. O Conselho de Defesa Sul-americano da UNASUL: criação institucional e cultura estratégica. Comparando a Defesa Sulamericana. Recife: Ed. UFPE.

Merke, Federico. 2004. Identidad y política exterior en teoría de las Relaciones Internacionales. Bs As: IDICSO, USAL.

Paradiso, José, y Luna Pont, Mariana. 2003. Paz y guerra en la trayectoria latinoamericana. Revista Universidad e Integracion, Estudios y Documentos sobre América Latina y el Caribe. Bs As: INTAL. 
Paradiso, José, y Sebesta, Lorenza. 2006. Interpretando el mundo. Desorden de la sociedad internacional y orden de los estados: la alternativa comunitaria. Revista Puente@europa. Nro o. Buenos Aires: Edic. Universitá di Bologna Representación.

Sondhaus, Lawrence. 2006. Strategic culture and Ways of War. New York: Routledge.

Tibiletti, Luis. 2009. La Construcción De La Identidad Estratégica Regional. Cuadernos De Actualidad En Defensa Y Estrategia. Nro 4. Bs As: Ed. Ministerio De Defensa.

Tibiletti, Luis. 2011. La Reformulación De Las Identidades Estratégicas Sudamericanas A Partir De Las Crisis Económicas. Ponencia Presentada. Xmo Congreso De La Saap Y Ucc. Argentina: Córdoba

Tokatlian, Juan Gabriel. 2010. La Autonomía Y La Política Mundial. In: Autonomía Y Neutralidad En La Globalización. Bs As: Edic. Capi.

Wallerstein, Immanuel, y Balibar, Etienne. 1991. Race, Nation And Class: Ambigous Identities. London: Ed. Verso.

Walt, Steven. 1991. The Renaissance Of Security Studies. International Studies Quaterly. Vol. 35. Nro 2. Junio 1991. 21 1-239.

Wendt, Alexander. 1994. Collective Identity Formation And The International State. The American Political Science Review. 88(2): 384-396. 


\section{Inovação, gestão e estruturação do Exército Brasileiro no Século XXI}

\section{Innovation, management and organization of XXI Century Brazilian Army}

FLÁVIO PIETROBON-COSTA*

\section{INTRODUÇÃO}

O Estado-Nação é uma entidade representativa e amalgamadora de uma sociedade: a Nação. Se o Poder Econômico e o Poder Militar estiverem desvinculados não há segurança para o Estado-Nação. Neste caso, esses Poderes conflitarão com as necessidades de integração entre a ação econômica e a segurança alcançada por uma força militar dissuasória.

Se estes Poderes não forem integrados o desenvolvimento social, o crescimento econômico, e suas sustentabilidades, bem como a preservação ambiental, estarão em risco. A sociedade estará em risco de perda de seus recursos, de sua integridade e de seu desenvolvimento. Estará vulnerável a oponentes externos ou aventureiros internos.

Os projetos de presente e futuro da respectiva sociedade, sua visão de futuro e planejamento estratégico, fornecem o eixo da ação econômica, de sustentabilidade de seu crescimento, de preservação ambiental, desenvolvimento social, e orientam a estratégia militar.

Os crescentes desafios para os Estados Nacionais no século XXI são tão diversos quanto o número de novos atores da geopolítica internacional, como os BRICS, Organizações Não Governamentais, ONGs, e Organizações Não Estatais de Interesse Privado, ONIPs (Wilson e Stupnytska 2007; Forbes 2008). Paralelamente assistimos à redução de poder dos Estados Unidos (Galbraith 1979; Kennedy 1988; Da Silva 2008), e à acelerada ascensão de poder da República Popular da China, ou Zhongguo (Muraqiek 2005), como potencial rival dos Estados Unidos (Fairban e Goldman 2008; Gilfford 2007)

$\mathrm{O}$ atual sistema político internacional tem interesses tão dispersos e di-

* Universidade Estadual de Santa Cruz, Depto. De Ciências Exatas e Tecnológicas, Ilhéus, BA. Professor adjunto A, DSc em Modelagem Computacional, MSc em engenharia Civil. Contatos: pietrobon.costa@pq.cnpq.br, flap@uesc.br; 73 3680.5057. 
fusos quanto o crescente número de atores majoritariamente não estatais. A maior parte destes, ONGs, ONIPs, Corporações Transnacionais, não é integrante de tratados e instituições reguladoras do sistema internacional de relações, que pudessem restringir ou controlar suas ações, usualmente conflituosas com interesses dos Estados - Nação.

O conjunto das reservas brasileiras de recursos naturais deverá ser, no futuro imediato, o gerador de recursos econômicos para o País. Pode, em conseqüência, vir a desempenhar o papel de solução para o antigo dilema "manteiga x canhões", i.e., uma possível divergência entre o atendimento às necessidades civis da população e da economia, e o atendimento às necessidades de preservação ambiental e de sustentabilidade de uso de recursos naturais, bem como de incorporação de avanços tecnológicos, na forma de investimentos em equipamentos e custos de manutenção das forças armadas, exigência necessária a uma potencia em crescimento e em processo de elevação de seus compromissos externos. As próprias forças armadas, na medida em que estejam bem equipadas, sob ação de um sistema de gestão de excelência, bem treinadas e motivadas, podem assumir a segurança de reservas de recursos naturais, assegurando o uso sustentável destes recursos e a preservação de parques e reservas do patrimônio natural brasileiro.

A $2^{a}$ parte deste artigo considera uma breve avaliação de valor econômico das reservas de recursos naturais brasileiros e suas implicações para a geoestratégia brasileira no século XXI. O item 3 aborda o papel chave das inovações como incorporação de diferencial de competitividade para o Exército Brasileiro. O item 4 considera uma proposta de balanceamento entre unidades militares pesadas, leves e de resposta rápida, e a implementação de paradigmas para o Exército Brasileiro: momentum - flexibilidade - controle - mobilidade - conhecimento, na forma de uma organização plenamente quaternária de suas brigadas.

\section{PREMISSAS GEOESTRATÉGICAS, A GESTÃO DE RECURSOS NATURAIS, E PERSPECTIVAS DA DEFESA TERRESTRE}

Se na Idade Média a mensuração de riqueza, e a fonte de onde emanava poder, era a extensão de propriedade de terra (Huberman 1986), em decorrência de ser o único sistema de produção significativo, a produção em massa, característica da atualidade, demanda uma pressão significativa sobre os recursos naturais do planeta, sejam renováveis ou de magnitude finita (Busby 2007; Asici 2013; Schilling e Chiang 2011). Recursos naturais 